\title{
Supporting Information for Publication: Glycan Cluster Shielding and Antibody Epitopes on Lassa Virus Envelop Protein
}

Suyong $\operatorname{Re}^{1,2, *}$ and Kenji Mizuguchi ${ }^{1,3,4, *}$

${ }^{1}$ Center for Drug Design Research, National Institutes of Biomedical Innovation, Health, and Nutrition, 7-6-8 Saito-Asagi, Ibaraki, Osaka 567-0085 Japan

${ }^{2}$ RIKEN Center for Biosystems Dynamics Research, Integrated Innovation Building 7F, 6-7-1 Minatojima-minamimachi, Chuo-ku, Kobe, Hyogo 650-0047, Japan

${ }^{3}$ Artificial Intelligence Center for Health and Biomedical Research, National Institutes of Biomedical Innovation, Health, and Nutrition, 7-6-8 Saito-Asagi, Ibaraki, Osaka 567-0085 Japan

${ }^{4}$ Institute for Protein Research, Osaka University, 3-2 Yamadaoka, Suita, Osaka 565-0871, Japan

*Corresponding Authors: suyongre@nibiohn.go.jp, kenji@nibiohn.go.jp

\section{Contents:}

Supporting Figures S1-S6 


\begin{tabular}{llll}
\hline & & & \\
\hline
\end{tabular}

Figure S1. Structure details of the glycans attached to GPC trimer models. 


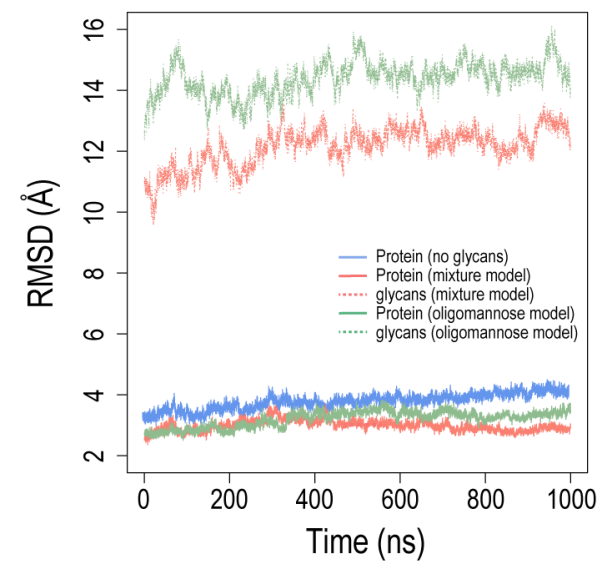

Figure S2. Structural convergence. Root mean-square deviations (RMSDs) of the protomer and glycans along the simulation time for GPC trimers with (red and green for the mixture and oligomannose models, respectively) and without glycans (blue). For the protomer (solid line), $\mathrm{C} \alpha$ atoms RMSDs were calculated for each of three protomers and averaged. The glycans' RMSDs (dashed line) were calculated for $\mathrm{C} 1$ atom of each saccharide molecule. 


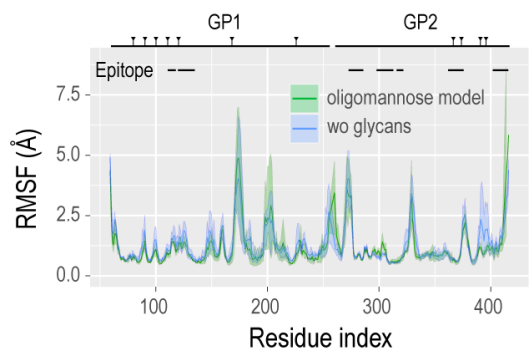

Figure S3. Protein fluctuation. C $\alpha$ atoms root mean-square fluctuations (RMSFs) of GPC trimers with (green, oligomannose model) and without (blue) glycans. The corresponding color shades show the deviation of RMSF values in three monomers. The positions of the glycosylation sites (vertical bar with inverse triangle) and know epitopes (horizontal bar) are shown for comparison. 


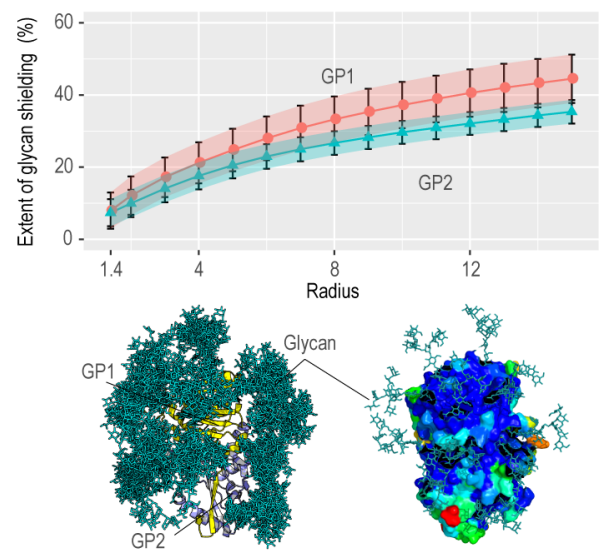

Figure S4. Glycan shielding. (top) Extent of glycan shielding in each of GP1 and GP2 domains estimated as a ratio of the reduction of solvent accessible surface area (SASA) upon glycosylation (oligomannose model) over SASA without glycans $\left.\left(\left(\mathrm{SASA}_{\mathrm{wo} / \mathrm{gly}}-\mathrm{SASA}_{\mathrm{wt} / \mathrm{gly}}\right) / \mathrm{SASA}_{\mathrm{wo} / \mathrm{gly}} \times 100\right)\right)$. The values are plotted along the probe radius used for SASA calculations. (bottom) Molecular representation of the glycan shielding by an overlay of 10 frames (every $100 \mathrm{~ns}$ ) of the simulation trajectory and the surface representation of per residue SASA values (blue to red for 0 to $576 \AA^{2}$ ). 


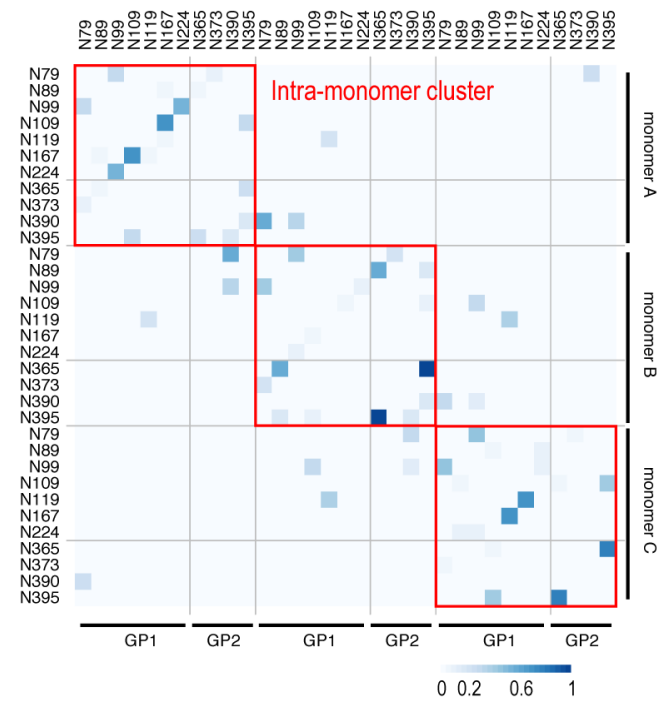

Figure S5. Glycan-glycan interaction. A contact map representation of glycan-glycan interaction probability having the distance between any heavy atoms for a pair of glycans $<5 \AA$ in the simulation. Red boxes highlight the intramonomer clusters. 

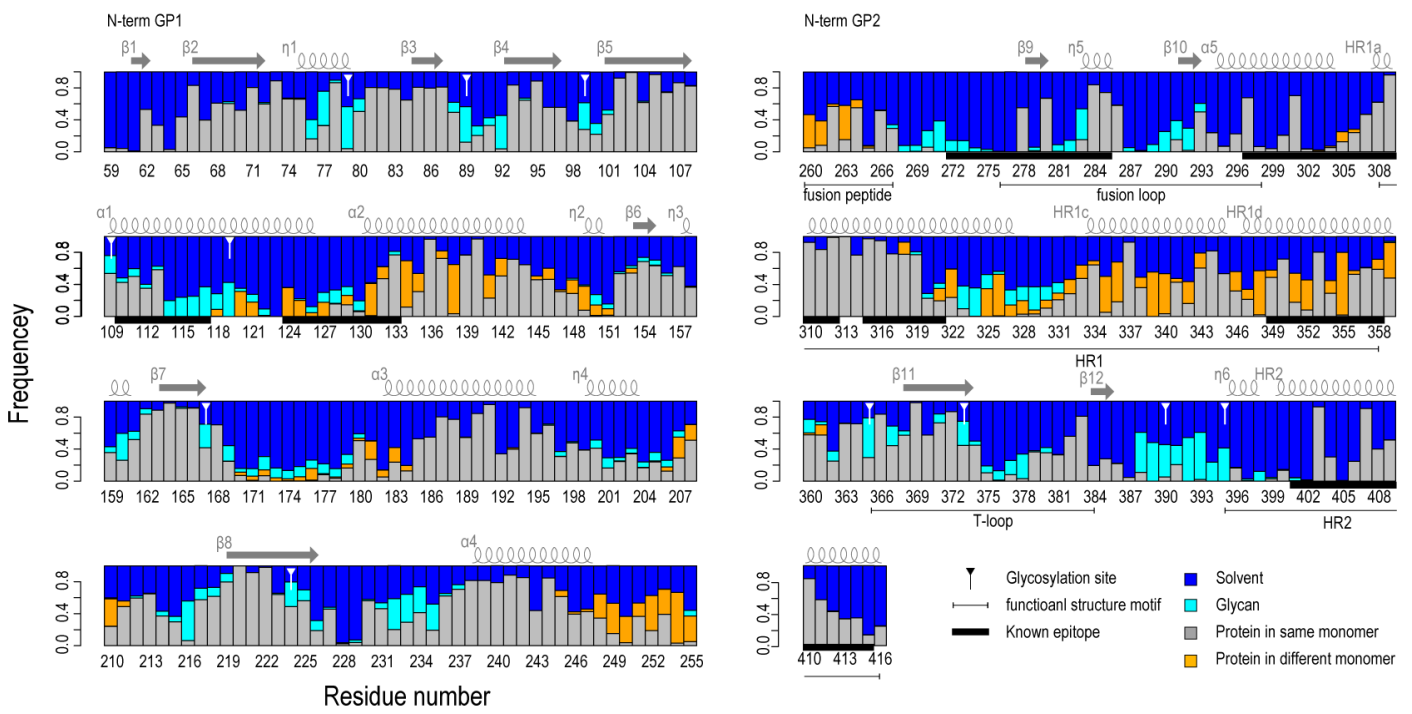

Figure 6. Glycan-protein interactions. Interaction pattern of GPC residues with their surroundings (other GPC residues, glycans, and water molecules). The interactions between a given residue and surroundings were calculated by counting the number of contacts in which the distance between any heavy atoms of an interaction pair is $<5 \AA$. The numbers were normalized for each interaction partner to get relative frequency. For each residue, the interaction frequencies for different partner are shown (blue: water; cyan: glycans; gray: other residues in the same monomer; orange: other residues in the different monomer). The locations of glycosylation sites, functional region, and known epitopes are also shown for comparison. 